Original Paper

\title{
Lack of Prenylated Proteins, Autophagy Impairment and Apoptosis in SH-SY5Y Neuronal Cell Model of Mevalonate Kinase Deficiency
}

\author{
Paola Maura Tricarico Alessandra Romeo $^{b}$ Rossella Gratton ${ }^{b}$ Sergio Crovella ${ }^{a, b}$ \\ Fulvio Celsia
}

aInstitute for Maternal and Child Health - IRCCS "Burlo Garofolo" - Trieste, bUniversity of Trieste, Trieste, Italy

\section{Key Words}

Mevalonate Kinase $\cdot$ Mevalonate Kinase Deficiency $\cdot$ Autophagy $\cdot$ Apoptosis $•$ LC-3 $・$ Rho-A $•$ Prenylation • GFP

\begin{abstract}
Background/Aims: Mevalonate Kinase Deficiency (MKD), is a hereditary disease due to mutations in mevalonate kinase gene (MVK). MKD has heterogeneous clinical phenotypes: the correlation between MVK mutations and MKD clinical phenotype is still to be fully elucidated. Deficiency of prenylated proteins has been hypothesized as possible MKD pathogenic mechanism. Based on this hypothesis and considering that neurologic impairment characterizes Mevalonic Aciduria (MA), the most severe form of MKD, we studied the effects of I268T and N301T MVK mutations on protein prenylation, autophagy and programmed cell death in SHSY5Y neuroblastoma cell lines. Methods: SH-SY5Y cells were transiently transfected, with the pCMV- 6 plasmid containing MVK wild type and the two mutated sequences. Protein prenylation levels were evaluated using GFP-RhoA-F to assess farnesylation, and GFP-RhoA to evaluate geranylgeranylation; autophagy was measured by evaluating LC3 and p62 protein levels, while Annexin V-FITC and Propidium Iodide staining allowed apoptosis detection. Results: MVK mutants' over-expression causes decreased levels of farnesylation and geranylgeranylation, and also increased LC3 lipidation in SH-SY5Y, with concomitant p62 accumulation. Treatment with bafilomycin A1 (an inhibitor of vacuolar H+-ATPase, a late autophagy inhibitor) further increase LC3-II and p62 levels, suggesting that degradation of autophagolysosome could be impaired. SH-SY5Y, with both MVK mutants, showed apoptosis increase; the presence of N301T associated with augmented cell death. Conclusions: We hypothesize that mevalonate pathway impairment causes alteration of farnesylation and geranylgeranylation proteins and alteration of the autophagic flux; these changes can induce apoptosis, possibly more relevant in the presence of N301T mutation.




\section{Introduction}

Mevalonate Kinase Deficiency (MKD) is an auto inflammatory and monogenic disease caused by mutations in mevalonate kinase gene (MVK, 12q24.11, NM_000431) that encodes for a key enzyme of mevalonate pathway, the Mevalonate Kinase (MK) protein (EC:2.7.1.36) (see Fig. 1a for complete description). MKD shows heterogeneous clinical phenotypes, with a milder form of the disease known as Hyper-IgD Syndrome (HIDS, OMIM \#260920) and a more severe one, the Mevalonic Aciduria (MA, OMIM \#610377).

MA patients are characterized by recurrent fever attacks $\left(>38.5^{\circ} \mathrm{C}\right)$, with skin rashes, hepatosplenomegalia, cutaneous rash, aphtae, arthralgia, abdominal pain with diarrhoea and vomiting and by critical neurologic phenotype with psychomotor retardation, hepatopathy and cerebellar ataxia $[1,2]$. More than $50 \%$ of MA patients die in infancy or early childhood and few of them survive to adolescence [3].

MVK genetic variants are known to be associated with MKD (at present 204 variations have been reported according to INFEVERS database, http://fmf.igh.cnrs.fr/ISSAID/ infevers/); however, MKD can shows great genotype-phenotype heterogeneity, as the same mutations could be associated both to severe and mild form of the disease, as observed for the I268T mutation [4-6]; moreover, there are other mutations specific just for the severe form, MA, such as N301T [7-9]. Genotype-phenotype heterogeneity could be related to the presence of other modifier genes that predispose or amplify deregulation of the mevalonate pathway in this disease $[10,11]$. Despite the various studies conducted in this direction, the role of these genes has yet not been clarified. Thus, the link between the genetic defects and MKD phenotypes remains to be elucidated.

The severity of MKD is correlated with MK enzymatic activity: if it drops below 1\% it leads to MA, while if its levels are between $1 \%$ to $7 \%$ it leads to the milder form of MKD [8]; unfortunately, the precise correlation between $M V K$ gene mutation and possible alterations in MK enzyme structure has not been completely explained. Recently, an article predicted the in silico effects of $M V K$ known mutations in the three-dimensional structure of the enzyme, identifying two hot spot regions (comprised between residues 8-35 and 234-338) for MA. These two regions are located around the inside of protein's cleft close to the active site and around the domain interface [12]. Therefore, variations of these amino acid residues, distorting the active site conformation, have been hypothesized as responsible for the low MK activity found in MA.

Mevalonate pathway is fundamental for the production of cholesterol and other molecules, such as farnesyl-pyrophosphate and geranylgeranyl-pyrophosphate, crucially involved in protein prenylation [13]. Protein prenylation is a key biochemical modification responsible for targeting specific proteins to cell membranes and activating specific protein for normal cell metabolism [13] (see Fig. 1a for complete description of the pathway). One of the major accredited MKD pathogenic hypothesis relies on the lack of prenylated proteins: the activation of two small GTPase (RhoA and Rac1), which requires geranygeranyl pyrophosphate, has been reported as disrupted in fibroblasts from MKD patients [14]. Van der Burgh and co-authors subsequently confirmed this pathogenetic hypothesis and expanded it, suggesting a strong link between shortage of prenylated proteins, mitochondrial damage and autophagy. In previous studies, we also observed a correlation between mevalonate pathway impairment, obtained by administration of the pathway blocker Lovastatin, and mitochondrial damage that induces an increase of apoptosis, in neuronal, microglial and monocytic cell lines [15-17].

Considering that the sole available findings on MKD pathogenesis used biochemical model [18] and no genetic model mimicking the effects of $M V K$ mutations in vitro has been proposed at present time, we established a transient expression model in neuroblastoma cell line (SH-SY5Y) using MVK coding plasmid carrying two different mutations: I268T (HGMD CM990888) and N301T (HGMD CM920489). A number of different mutations have been associated either with HIDS or MA, but we decided to focus our attention on 


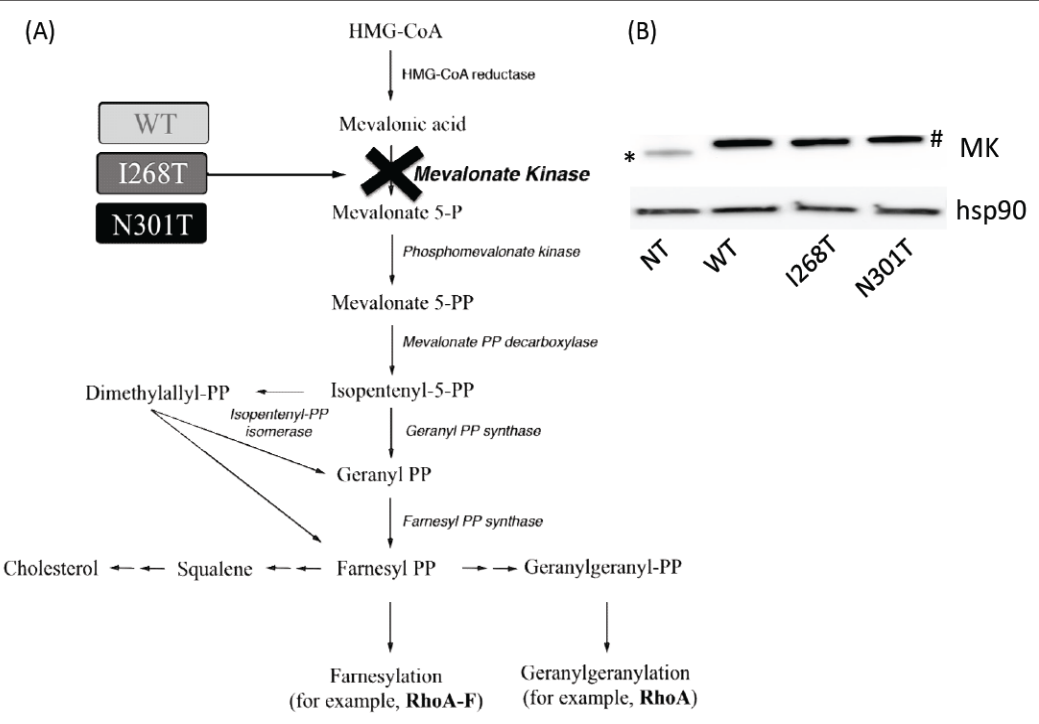

Fig. 1. Mevalonate kinase pathway and transient expression model of MKD. (a) Schematic representation of the mevalonate pathway. In vitro MKD transient expression model, was established using $M V K$ coding plasmid carrying two different mutations: I268T (orange), and N301T (red) and WT (Wild-Type of $M V K$, yellow). The principal product of mevalonate pathway is cholesterol, but this metabolic pathway is also important for the production of farnesyl-PP and geranylgeranyl-PP that generates respectively farnesylation and geranylgeranylation of small GTPase, among other proteins. (b) Levels of MK protein with western blot in SH-SY5Y cells carrying WT MVK or mutant alleles (I268T and $\mathrm{N} 301 \mathrm{~T}$ ). MK has been detected with policlonal antibody: it is possible to distinguish between exogenous (\#) and endogenous $\left(^{*}\right.$ ) MK based on molecular weight, being the exogenous MK fused with MYC peptide (already present in the original plasmid used for transfection) and consequently heavier ( $43.5 \mathrm{KDa})$ than the endogenousone ( $42 \mathrm{KDa}$ ) Hsp90 is the reference protein.

these two mutations since both localized in the in silico predicted hot spot region for MA [12]. Furthermore, I268T, when present in homozygosity is associated with MA, while in heterozygosity could be linked to HIDS; it is also the second-most frequent mutation associated with MKD. N301T instead, is specifically associated to MA, with an undetectable level of enzymatic activity. All this considered we evaluated the effects of MVK mutants' overexpression in SH-SY5Y neuronal cell line focusing on the important neuronal impairment typical of MA patients by analysing protein prenylation levels, autophagic flux and cell death.

\section{Materials and Methods}

\section{Cell Culture}

SH-SY5Y neuroblastoma cells were chosen considering that the more severe clinical signs characterizing MA (the most severe phenotype of MKD) involve the nervous system; moreover SH-SY5Y cells are a widely used model for neurodegenerative disease, possess neuronal features and are easy to transfect.

SH-SY5Y, kindly provided by Prof. S. Gustincich (Department of Neurobiology, International School for Advanced Studied S.I.S.S.A.-I.S.A.S. Trieste, Italy) were cultured in 44.5\% MEM/EBSS (Euroclone, Italy), 44.5\% HAM'S/F12 (Euroclone, Italy), supplemented with 10\% fetal bovine serum (FBS, Euroclone, Italy), non-essential amino acid solution $1 \times$ (NEAA, Euroclone, Italy), $2 \mathrm{mM}$ glutamine and penicillin streptomycin amphotericin B $1 \times$ solution (Sigma Chemical Co. Aldrich St. Louis, MO) and used between passages 4 to 10 to avoid cellular senescence. Transfection was performed using Lipofectamine (Life Technologies, Carlsbad, USA) following manufactures' protocols. When co-transfection was performed, MVK and GFP-RhoA or GFPRhoAF plasmids were used in a 10:1 ratio, to minimize the chance to have cells with only one plasmid. To assess impairment of autophagic flux, cells were treated with Bafilomycin A1 (Sigma Chemical Co. Aldrich St. Louis, MO) $5 \mathrm{nM}$ for $16 \mathrm{~h}$ then processed for western blot. 


\section{Cellular Physiology Cell Physiol Biochem 2017;41:1649-1660 \\ \begin{tabular}{ll|l} 
and Biochemistry Published onIIne: IVarch 28, 2017 & $\begin{array}{l}\text { (c) 2017 The Author(s). Published by S. Karger AG, Basel } \\
\text { www.karger.com/cpb }\end{array}$
\end{tabular} \\ Tricarico et al.: Prenylated Proteins and Autophagy in MKD}

Plasmids and site directed mutagenesis

pCMV-6 MVK-expressing plasmid (Wild Type; RefSeq: NM_000431.1) (WT) was obtained from Origene (Rockville, USA) (RC201971). On this template, two mutations in the coding sequence were obtained: I268T and N301T, using the QuikChange site-directed mutagenesis kit (Stratagene, La Jolla, California). Primers were designed using the "PrimerX" web application (http://www.bioinformatics.org/primerx/index.htm). Mutagenesis was confirmed by direct sequencing. G. Del Sal (LNCIB, Trieste, Italy) kindly provided plasmids GFP-RhoA and GFP-RhoAF, generated from pLPC.

\section{GFP intracellular distribution}

Intracellular distribution of GFP-RhoA and GFP-RhoAF was evaluated using Nikon C2+ confocal microscope. Briefly, cells were grown on glass coverslip, transfected with the different plasmids and then stained with Vybrant ${ }^{\circledast}$ DiD cell-labeling solution (ThermoFisher) following manufacturer's indications. After 20 minutes incubation cells were fixed in 4\% paraformaldehyde and imaged using 63X objective: 10-15 cell-images for each experiment were taken. Cells were acquired as stack of 20 images with a depth of field of $10 \mu \mathrm{m}$ and each image was separated by $0.5 \mu \mathrm{m}$. Using the 'Z-Stack projection' plugin of Fiji Software [17] images stack were averaged to a single two-dimensional image. For GFP-RhoA and GFPRhoAF parallel images for nuclear staining (DAPI; Life Technologies) were obtained. Composite images formed by GFP, Vybrant ${ }^{\circledR}$ DiD and DAPI signals were made using Fiji software [19] and overlap between the GFP and DAPI signals was evaluated using JACoP plugin [20]; if overlap coefficient was $>0.60$, intracellular distribution was considered "cytoplasmic". The reference value for overlap coefficient was obtained using cells transfected only with GFP-RhoA or GFP-RhoA-F plasmid without $M V K$, called not treated (NT): this mean value corresponds to the basal overlap coefficient between the two signals.

\section{Western Blot}

SH-SY5Y cells overexpressing MVK, either WT or I268T or N301T, were lysed in RIPA buffer $(10 \mathrm{mM}$ Tris-HCl pH 8.0, 1 mM EDTA, 1\% Triton X-100, $140 \mathrm{mM} \mathrm{NaCl}$ ). Cellular lysates were quantified using Bradford assay (Biorad, Hercules, USA); equal amounts of protein were loaded on 4-20\% Tris-Tricine gels (Biorad) and transferred on nitrocellulose membrane (Biorad, Hercules, USA). Membranes were then incubated with primary antibodies and then developed with HRP-conjugated secondary antibodies and Clarity ${ }^{\mathrm{TM}}$ substrate (Biorad Hercules, USA). Primary antibodies were: anti-MVK (Origene, Rockville, USA; TA313341); antihsp90 (SantaCruz, Dallas, USA); anti-LC3 (Sigma Chemical Co. Aldrich St. Louis, MO), anti-p62 (SantaCruz, Dallas, USA).

\section{Apoptosis analysis}

Apoptosis was evaluated in SH-SY5Y cells expressing pCMV-6 plasmid with MVK mutations, using flow cytometry (Annexin V-FITC Apoptosis Detection Kit, Immunostep, Spain). Fluorescence was acquired with CyAn ADP analyser and Summit software (Beckman Coulter, Brea, CA, USA), then analysed with FlowJo software (version 7.6, Treestar, Inc., St Ashland, OR, USA).

\section{Statistical Analysis}

Statistical significance was calculated using one-way ANOVA with Bonferroni post hoc test correction. Analyses were performed using GraphPad Prism software (version 5.0, GraphPad Software, Inc., La Jolla, CA, USA).

\section{Results}

\section{Mutant MK expression}

We obtained two mutated MK versions using site-directed mutagenesis on plasmid carrying $M V K$ wild-type cDNA sequence. Giving the fact that MK mutated proteins could have different stabilities (half-life), as a first step, we assessed protein levels in SH-SY5Y cells carrying MVK WT or mutant alleles (I268T and N301T). As shown in Fig. 1b, SH-SY5Y cells showed homogeneous increase of MK protein levels independently of the genetic variants carried, thus demonstrating that all variants have similar stability. 


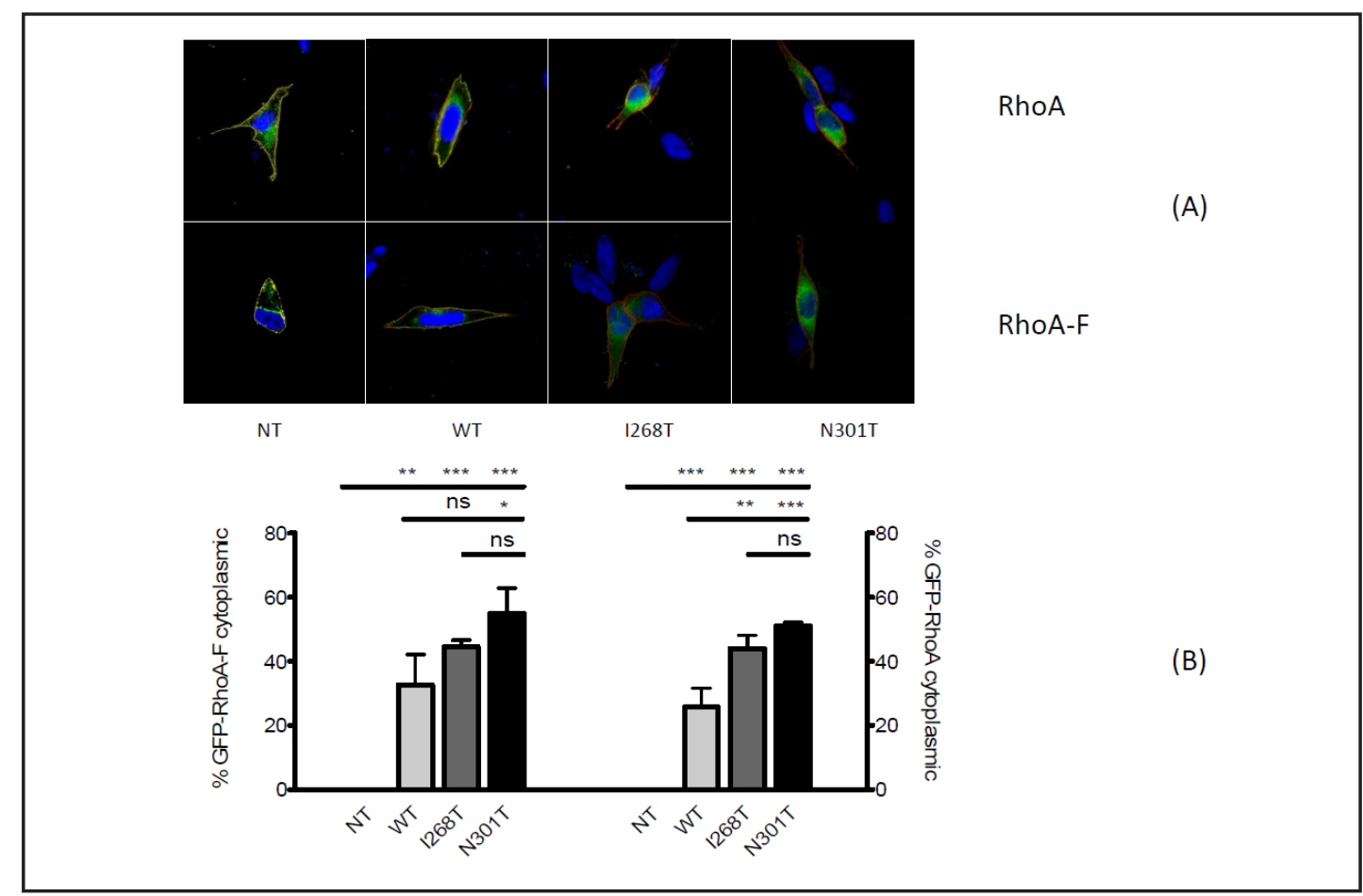

Fig. 2. Mutations in $M V K$ causes alterations of farnesylation and geranylgeranylation of proteins. (a) SH-SY5Y transiently transfected with GFP-RhoA-F or GFP-RhoA. GFP-RhoA-F with a farnesylation consensus sequence is sensitive to farnesylation; indeed, GFP-RhoA with a geranylageranylation consensus sequence is sensible to geranylgeranylation. Representative images are shown. In red, membrane staining with Vybrant ${ }^{\circledR}$ DiD. I268T and N301T demonstrate the cytosolic distribution. Scale bars, $10 \mu \mathrm{m}$. (b) Percentages of cells with cytosolic distribution of GFP-RhoA-F and with cytosolic distribution of GFP-RhoA according to $M V K$ sequences carried are reported. Data are derived from $\mathrm{n}=3$ independent experiments; $10-15$ cell images for each experiment were taken. $\mathrm{NT}=$ not transfected cells, WT $=$ Wild-Type $M V K, \mathrm{I} 268 \mathrm{~T}=M V K$ mutated I268T, N301T=MVK mutated N301T. Statistical significance was calculated using one-way ANOVA with Bonferroni post hoc test correction. ${ }^{*} \mathrm{P}<0.05$; $* * \mathrm{P}<0.01, * * * \mathrm{P}<0.001$, ns: not significant.

Protein prenylation levels assessment

Considering that one of the demonstrated effects of mevalonate pathway alteration, also caused by mutated MK, is the diminished levels of prenyl-groups, specifically farnesylpyrophosphate and geranylgeranyl-pyrophosphate, necessary for secondary modifications of specific proteins, we determined the effect of MVKI268T and N301T mutations on proteins prenylation. To this aim, we co-expressed either WT or mutant proteins together with two different fluorescent sensors, specifically GFP-RhoA-F to assess farnesylation, and GFP-RhoA to evaluate geranylgeranylation.

Although novel, this technique has been previously validated as shown in the article of Sorrentino and co-authors [21] and it is a widely accepted methodology to assess protein prenylation levels.

As shown in Fig. 2a, GFP-RhoA-F or GFP-RhoA alone, in NT condition, localized in cellular membranes, most prominently in perinuclear region, this localization is also confirmed by co-localization with Vybrant DiD plasma membrane staining and GFP. Instead, in transfected cells with $M V K$, GFP-RhoA-F or GFP-RhoA the localization is considered cytoplasmic; in fact, it is diffused into cytosol and not only in cellular membranes; moreover plasma membranes are mainly marked with Vybrant DiD.

In order to obtain an unbiased measure, we decided to use an objective parameter to quantify the number of cells possessing a cytoplasmic localization of these GFP- based

\section{KARGER}




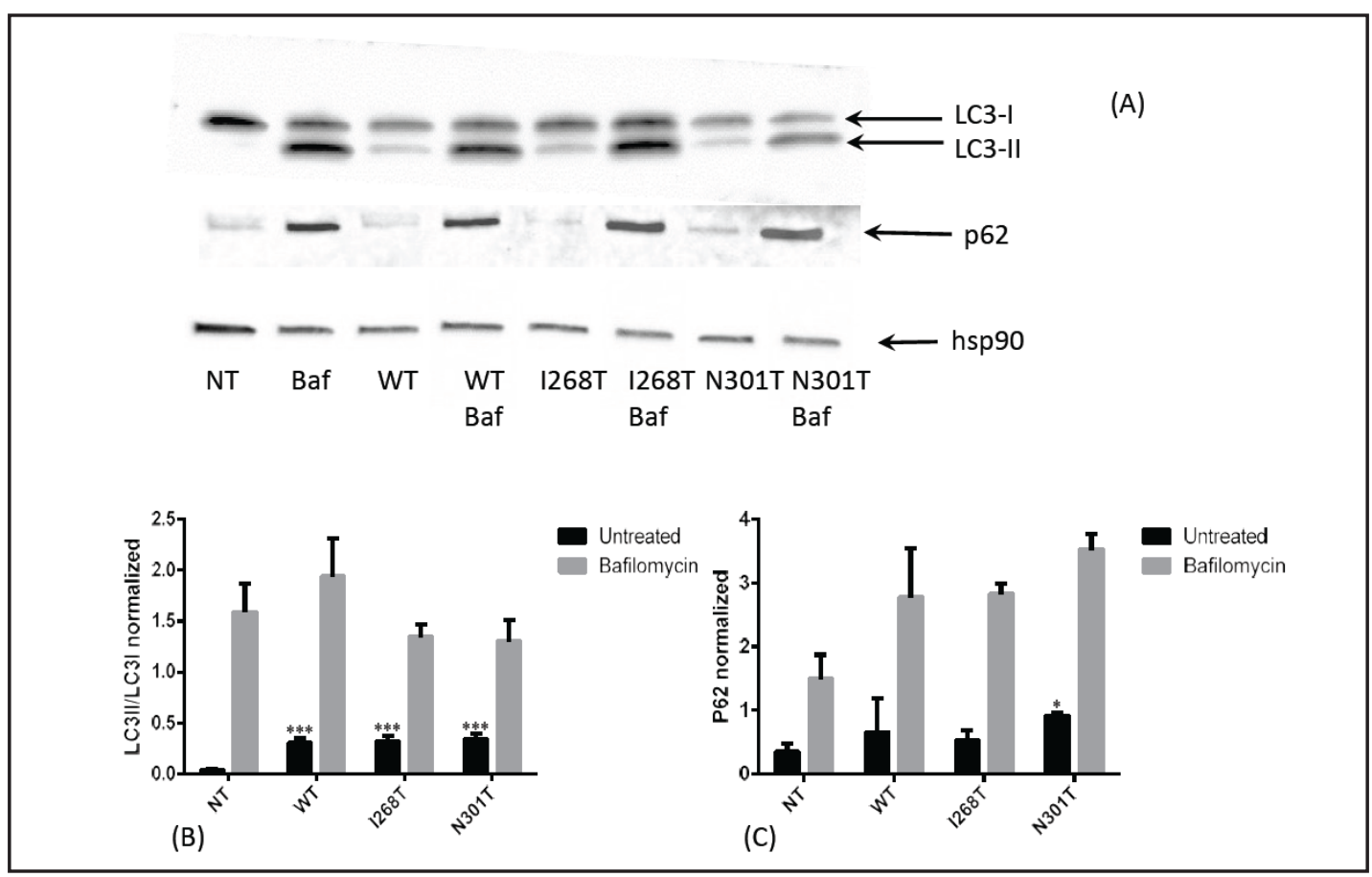

Fig. 3. $M V K$ mutations induce defective autophagy. (a) Representative Western blots showing expression of LC3II, LC3-I, p62 and HSP90, the latter used as the reference protein. NT= not transfected cells, WT=Wild-Type $M V K$, I268T $=M V K$ mutated I268T, N301T $=M V K$ mutated N301T, Baf $=$ Bafilomycin A1 treatment. (b) Quantification of LC3-II increase, using LC3-II/LC3-I optical density ratio, ${ }^{* * *} \mathrm{P}<0.001 \mathrm{vs} \mathrm{NT}$, one-way ANOVA with Bonferroni post hoc test correction. (c) Quantification of p62 increase, normalized to HSP90 optical density. Statistical significance was calculated using one-way ANOVA with Bonferroni post hoc test correction. $* \mathrm{P}<0.05$; , ns: not significant.

sensor: the co-localization coefficient, obtained using overlap of the blue (DAPI) signal with the green (GFP) signal. This coefficient (see materials and methods) was obtained using FiJi, software for image analysis $[17,18]$. We used as a baseline the coefficient obtained using cells transfected only with GFP-RhoA or -RhoAF, hypothesising that this would represent the "normal" distribution of these sensors. As a positive control, we treated cells with Lovastatin, obtaining a mean co-localization coefficient of almost 1, representing complete co-localization (data not shown). After setting up the conditions, we quantified the number of cells with a cytoplasmic localization for each condition. After transfecting with MVK I268T and N301T, we observed a significant increase of the number of cells with GFP-RhoA-F and GFP-RhoA cytoplasmic localization (\%GFP-RhoA-F cytoplasmic: I268T $44.54 \pm 2.11, \mathrm{p}<$ 0.001 ; N301T $54.94 \pm 7.92, \mathrm{p}<0.001$ ) (\%GFP-RhoA cytoplasmic: I268T $44.10 \pm 0.07, \mathrm{p}<$ 0.001 ; N301T $51.19 \pm 1.08, \mathrm{p}<0.001)$ when compared with NT condition (\%GFP-RhoA-F and \%GFP-RhoA cytoplasmic: NT:0) (Fig. 2b); in SH-SY5Y carrying N301T mutation, we detected a significantly increase of cells with GFP-RhoA-F and GFP-RhoA cytoplasmic localization if compared with WT (\%GFP-RhoA-F cytoplasmic: WT $32.79 \pm 9.39, \mathrm{p}<0.01$ ) (\%GFP-RhoA cytoplasmic: WT $25.75 \pm 5.87, \mathrm{p}<0.001$ ); instead in SH-SY5Y with I268T mutation the increase becomes significant only for the GFP-RhoA localization when compared to WT (Fig. 2b).

\section{Autophagic flux disarrangement}

Autophagy is a well-coordinated process that contributes to the removal of unused or damaged proteins and organelles [22,23]; autophagy is regulated by a variety of proteins, and numerous GTPases intervene in this mechanism. One of the activation mechanisms of GTPase is prenylation, necessary to move these proteins into membranes, where they 


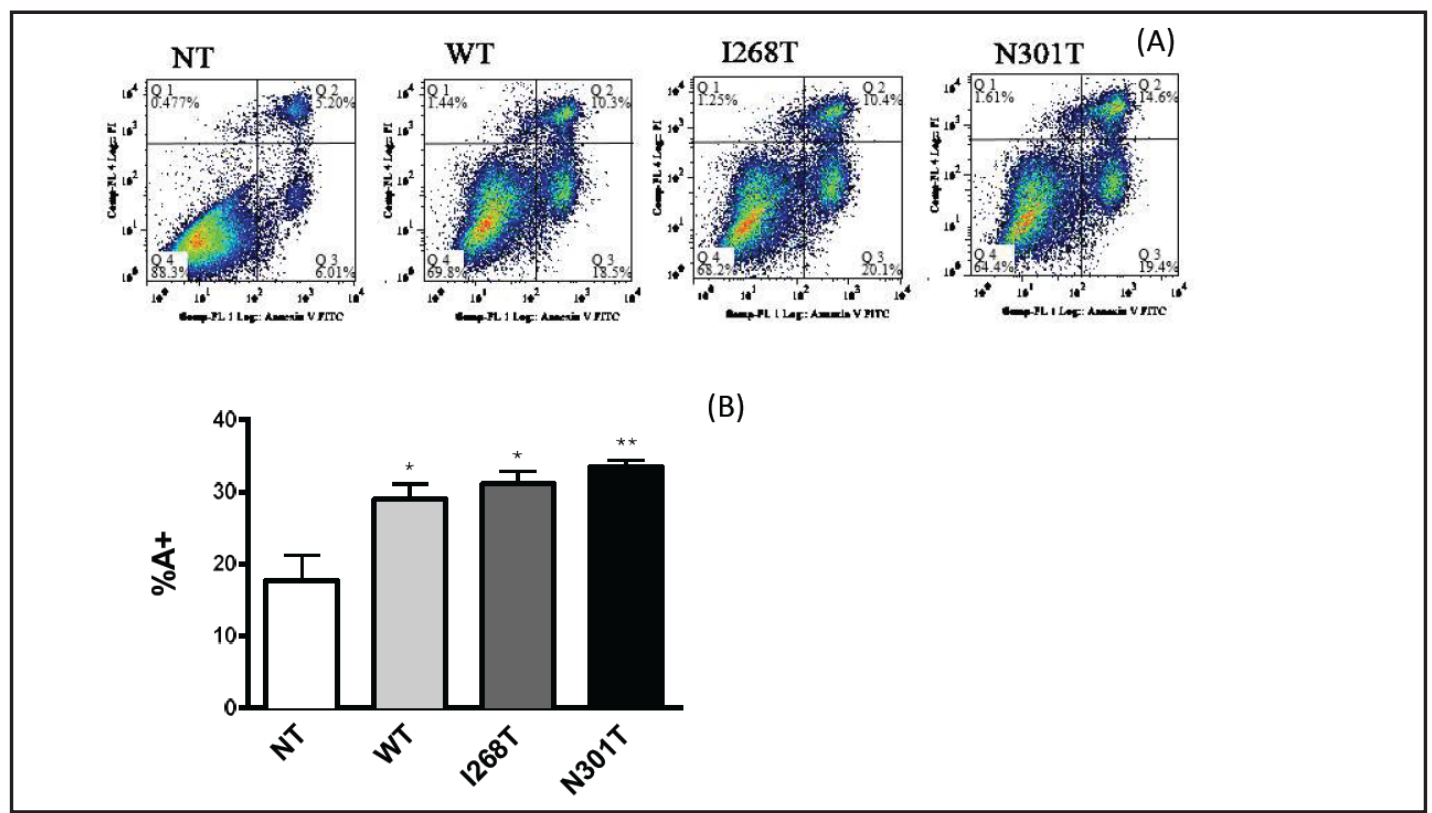

Fig. 4. MVK mutations cause apoptosis in SH-SY5Y. (a) Representative flow cytometry plots of SH-SY5Y cells carrying WT MVK or mutant proteins (I268T and N301T) stained with Annexin V and Propidium Iodide. Dot plots show the staining with Annexin V FITC and Propidium Iodide after gating on FSC/SSC to exclude debris. Numbers in the corners of quadrants represent the percentage of the total. (b) Quantification of apoptotic cells, showing the average of $\%$ Annexin positive cells $(\% \mathrm{~A}+) \pm$ standard deviation (S.D.) of three independent experiments. NT= not transfected cells, WT=Wild-Type $M V K$, I268T $=M V K$ mutated I268T, N301T=MVK mutated N301T. Statistical significance was calculated one-way ANOVA with Bonferroni post hoc test correction. $* \mathrm{P}<0.05$; $* * \mathrm{P}<0.01$, ns: not significant.

exert their functions. After observing that MVK I268T and N301T alter prenylation levels, we decided to investigate if the presence of MK mutants could also modify the autophagic process in SH-SY5Y.

As a marker of the autophagy initiation, we analysed the ratio between the lipidated (active) form of LC3 (LC3-II) and the non-lipidated moiety (LC3-I). As shown in Fig. 3a, the presence of mutated MK significantly increases LC3 lipidation (LC3II/LC3I normalized: WT $0.305 \pm 0.044, \mathrm{p}<0.001 ; \mathrm{I} 268 \mathrm{~T} 0.319 \pm 0.056, \mathrm{p}<0.001 ; \mathrm{N} 301 \mathrm{~T} 0.341 \pm 0.057, \mathrm{p}<0.001$ ) if compared to NT (LC3II/LC3I normalized: NT $0.037 \pm 0.017$ ), thus activating autophagic process. Treatment with Bafilomycin $\mathrm{A} 1$, an inhibitor of vacuolar $\mathrm{H}^{+}$-ATPase, responsible for fusion between autophagosomes and lysosomes, further increases the ratio LC3-II/LC3-I (LC3II/LC3I normalized: Baf 1.59 \pm 0.282; WT+Baf $1.94 \pm 0.371$; I268T+Baf $1.349 \pm 0.119$; $\mathrm{N} 301 \mathrm{~T}+\mathrm{Baf} 1.303 \pm 0.209$ ), (Fig. $3 \mathrm{~b}$ ) demonstrating that the first part of autophagic process is still functioning.

Sequestosome-1/p62 is a key protein further downstream of autophagic process interacting with LC3-II and other proteins to target them for degradation and it is itself degraded by autophagy; its levels are useful to monitor autophagy.

We observed that p62 levels increased in SH-SY5Y cells with expression of MVK WT and I268T and significantly augmented in N301T mutants (p62 normalized: WT $0.656 \pm 0.308$, ns; I268T $0.52 \pm 0.093$, ns; N301T $0.916 \pm 0.027, \mathrm{p}<0.05$ ) if compared with NT (p62 normalized: NT $0.344 \pm 0.074$ ) (Fig. 3a and Fig. 3c). Simultaneous treatment with Bafilomycin A1 further augmented p62 expression (p62 normalized: Baf $1.497 \pm 0.216$; WT + Baf $2.778 \pm 0.444 ; \mathrm{I} 268 \mathrm{~T}+$ Baf $2.832 \pm 0.097 ; \mathrm{N} 301 \mathrm{~T}+$ Baf $3.527 \pm 0.141$ ). So it is possible to speculate that MK mutants impair autophagic process, further downstream of the fusion between autophagosomes and lysosomes, because p62 not degraded and co-treatment with Bafilomycin A1 further increases the expression of this protein. 


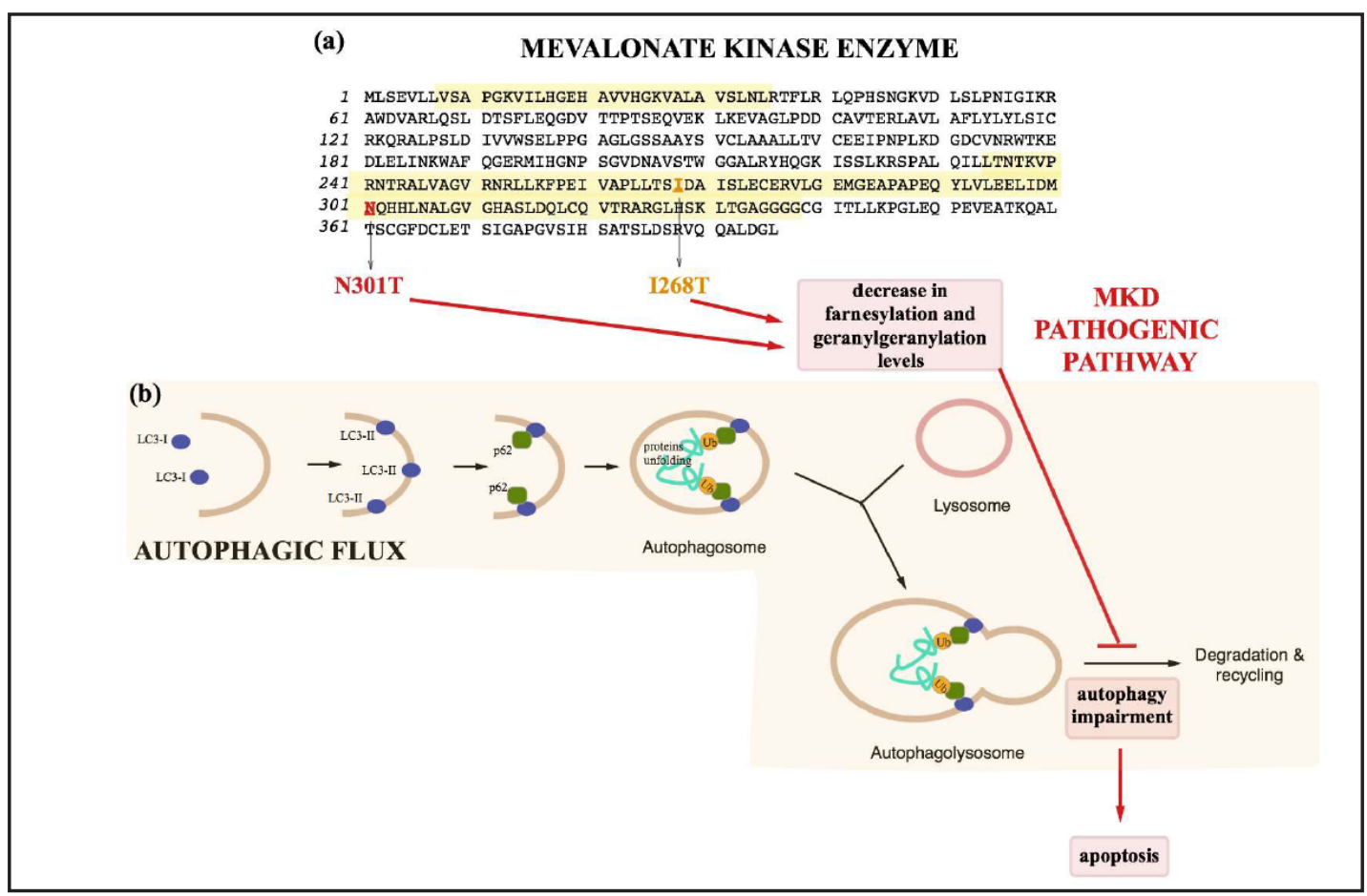

Fig. 5. Mutation hot spot regions in mevalonate kinase enzyme and MKD pathogenic pathway observed. (a) the hot spot regions (comprised between residues 8-35 and 234-338, highlighted in yellow) in mevalonate kinase (MK) enzyme sequence are schematically represented. The two mutations involved in the study are depicted in the sequence of MK: I268T (orange), and N301T (red). (b) autophagic flux is represented: LC3-I (non-lipidated) is converted into lipidated (active) form of LC3 (LC3-II); LC3-II mediates membrane elongation by associating with autophagosomal membrane. Adaptor protein p62 directly binds to ubiquitinated (Ub) proteins and acts as a receptor for Ub proteins. Subsequently, the autophagosome fuses with the lysosome to form an autophagolysosome. Finally all the proteins are degraded in the autophagolysosome and recycled. In red boxes the possible MKD pathogenic pathway is depicted: $M V K$ mutations (I268T and N301T) result in decrease in farnesylation and geranylgeranylation levels. Decrease of prenylated proteins induces autophagy impairment causing apoptotic cell death.

\section{Apoptosis}

Autophagy impairment is responsible for activation of apoptotic cell death in different disease models, specifically of neurodegenerative diseases [24-26]. It was then investigated if apoptotic cell death is modulated in SH-SY5Y transfected with MVK WT or I268T or N301T mutations.

Overexpression of MK mutants provoked an increase of apoptotic cell death; these results are also graphically evident in flow cytometric plots (Fig. 4a): the effect of transfection with $M V K$ (WT, I268T or N301T mutations) on apoptosis can be clearly observed in the upper and lower right quadrants (Q2 and Q3 respectively) of dot plot compared with the same quadrants of dot plot in NT condition. Of note, the overproduction of MK protein in WT caused augmented apoptosis (\%A+: WT $29.06 \pm 4.11, \mathrm{p}<0.05)$ with respect to NT conditions $(\% A+$ : NT $17.76 \pm 6.01)$; we also observed a significant increase of apoptosis in the presence of I268T and further increase in N301T (\%A+: I268T 31.17 $\pm 2.95, \mathrm{p}<0.05$; N301T $33.57 \pm$ $1.40, \mathrm{p}<0.01$ ) (Fig. 4b).

\section{Discussion}

Mevalonic Aciduria (MA), a severe form of MKD characterized by neurologic impairment, is caused by mutations at $M V K$ gene that encodes for MK enzyme. The genotype/phenotype 
correlation is not completely known and the entire pathogenic mechanism at the basis of the disease is only hypothetical, strongly needing to be experimentally unravelled. This lack of experimental evidence for genotype/phenotype correlation is mainly due to the absence of viable and reproducible models (either animal or cellular) useful to mimic the impact of $M V K$ mutations on the etiopathogenesis of MKD.

$M V K$-null cells are not viable: either $M V K$-KO or double dose $M V K$ mutation in homozygosis or compound heterozygosis is not compatible with cell life. Silencing technology has been also used in the attempt to obtain MVK-null cells [27] but no positive results have been achieved, having been able only to partially silencing $M V K$ (around $40 \%$ of efficiency). Several researchers tried to obtain a MVK KO mouse strain to mimic the effects of the mutations in animal model, but MVK-/- mice are not viable (they die in early embryonic stages) $[28,29]$. We also tried to introduce the mutations such as I268T and N301T, using CRISPR/Cas 9 system, but it has been impossible to obtain viable MVK null/null cells. At present, the Lovastatin driven biochemical block of mevalonate pathway has given the most exhaustive findings on the impairment of this pathway in cell models $[15,17]$.

All this considered, we tried to unravel the effect of selected $M V K$ mutation (I268T, associated to mild and severe MKD, and N301T specifically associated to MA) using a transient expression model in neuronal cell line (SH-SY5Y).

Our results suggest that mevalonate pathway impairment, due to these two MVK mutations, causes decreased levels of farnesylation and geranylgeranylation, which theoretically may trigger the impairment of autophagy. In our neuronal cell model, N301T mutation associated to MA, leads to a decrease in prenylated protein level, compared to I268T mutation associated to mild/severe MKD. This could prime an additional impaired autophagy, deduced by increased LC3 lipidation and increase of p62 levels in the presence of N301T mutation in comparison to I268T mutation; this apparent block of autophagy could be indeed cause of increased apoptosis (greater in the presence of N301T mutation when compared to I268T). We can hypothesize that the incremented apoptosis observed in the presence of N301T mutation could be involved, at least in part, with the severe neuronal phenotype observed in MA patients [30].

When considering the impact of I268T and N301T MVK mutations we observed that $\mathrm{N} 301 \mathrm{~T}$, associated with MA, showed a more severe response for all observed variables if compared to I268T mutation associated to severe/mild MKD (Fig. 5a). Both mutations occur in the in silico predicted hotspot regions for MA (Fig. 5b and Fig. 1a in Browne et al. [12]), and they both localizes in $\alpha$-helices at the dimer interface of MK enzyme [12]. It is possible to speculate that the two mutations alter in different manner the enzyme dimerization; however, there are no molecular dynamics data elaborating the effect of $M V K$ mutations on the ability of MK to dimerise.

The crosstalk between autophagy and apoptosis pathways has been demonstrated in different models [31]. Recently, in traumatic brain injury model, it was found that markers of increased and blocked autophagic flux co-localize with activated caspase-3. These results suggest that autophagy blockade could lead to apoptosis [32].

Autophagy is a complex process, multiple pathways are known to regulate and control this specific mechanism also with the intervention of numerous small GTPases, (for a complete review, see [33]) necessitating prenylation to correctly function. Although using a transient knock-in $M V K$ genetic model, our study suggests a tight link between impairment in autophagic flux and mevalonate pathway dysfunction, followed by cell death [34]. A recent work in patients with rheumatoid arthritis demonstrated that increasing autophagic flux could be beneficial toward lowering markers of inflammation [35] and a previous study also demonstrated that everolimus (inhibitor of mTor pathway) treatment, concomitant with methotrexate, could improve symptoms of patients suffering of rheumatoid arthritis [36]. Van der Burgh and co-authors have previously shown that treatment of MKD patients' monocyte with rampamycin, inhibitor of mTor pathway, slightly diminish IL-1 $\beta$ secretion after LPS challenge, although not to levels similar to the control [37]. These data showed how autophagy is a complex process and other possibly involved molecules need to be 


\section{Cellular Physiology Cell Physiol Biochem 2017;41:1649-1660

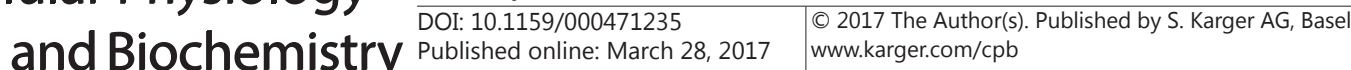 \\ Tricarico et al.: Prenylated Proteins and Autophagy in MKD}

evaluated. Furthermore, our findings support the key role of autophagy in MKD suggesting that mevalonate pathway disturbances could be responsible for autophagic flux blockade.

A recent study indicates that the Rab family of GTPase is not involved into release of IL-1 $\beta$ from MKD patients monocytes after an high temperature stimulus [38]. Rab GTPases necessitate of geranylgeranyl post-translational modification to be attached to membranes and then activated; thus these results further confirm our findings on the important role of farnesylation and not only geranylgeranylation in MKD pathogenesis. So, both prenylation pathways could be simultaneously involved, causing the decrease of active GTPases.

Moreover, Veluthakal and co-authors demonstrated that metabolic disturbances, specifically ER stress and mevalonate pathway blockade might cause accumulation of unprenylated trimeric-G-proteins. This appears to be due to the caspase- 3 activation that degrades farnesyl and geranylgeranyl transferase cleaving a-subunit of farnesyl and geranylgeranyl transferase [39]. This work demonstrates how metabolic stress could affect prenylation pathway, generating a wide cellular response.

We are aware that our study, having been performed using a $M V K$ mutated transient genetic model has various limitations, being over-expression of the target protein the most important one. This could lead to damages independently of the mutations, being MK WT, I268T and N301T all overexpressed on the same level and all causing increase on programmed cell death. In fact, it is thus possible that cholesterol pathway is altered simply by the enzyme increased levels, thus leading to apoptosis; however, our results using prenylation-sensitive GFP demonstrate that $M V K$ mutants have a different effect, significantly reducing the levels of prenylated proteins (see Fig. 2b). So, the relative small differences observed in apoptotic cell death between WT and mutant $M V K$ (see Fig. $4 \mathrm{~b}$ ) could be due to a confounding effect in which overexpression of MVK "masks" the effect of mutants by increasing the tendency to apoptosis. All this considered we have to cautiously consider the effects of $M V K$ transient mutations on apoptosis.

Taken together our results indicate a possible correlation between mevalonate pathway alteration, decrease of prenylated proteins levels, defective autophagy and apoptosis (Fig. 5).

To date, MKD is still an "orphan" disease, treatment is only supportive and there is no evidence-based therapeutic protocol. It will then be crucial to understand MKD and MA pathogenic mechanism in order to identify novel proteins involved, to the final aim of identifying effective therapeutic targets.

\section{Acknowledgements}

This study was supported by a grant from the Institute for Maternal and Child Health IRCCS "Burlo Garofolo" - Trieste, Italy (RC 42/2011).

\section{Disclosure Statement}

The authors declare no conflict of interest.

\section{References}

1 Drenth JP, Cuisset L, Grateau G, Vasseur C, van de Velde-Visser SD, de Jong JG, Beckmann JS, van der Meer JW, Delpech M: Mutations in the gene encoding mevalonate kinase cause hyper-IgD and periodic fever syndrome. International Hyper-IgD Study Group. Nat Genet 1999;22:178-181.

-2 Houten SM, Romeijn GJ, Koster J, Gray RG, Darbyshire P, Smit GP, de Klerk JB, Duran M, Gibson KM, Wanders RJ, Waterham HR: Identification and characterization of three novel missense mutations in mevalonate kinase cDNA causing mevalonic aciduria, a disorder of isoprene biosynthesis. Hum Mol Genet 1999;8:1523-1528. 


\section{Cellular Physiology Cell Physiol Biochem 2017;41:1649-1660 \begin{tabular}{ll|l} 
and Biochemistry Published onlIne: IVIarch 28, 2017 & $\begin{array}{l}\text { (C) } 2017 \text { The Author(s). Published by S. Karger AG, Basel } \\
\text { www.karger.com/cpb }\end{array}$
\end{tabular}}

- 3 Hoffmann G, Gibson KM, Brandt IK, Bader PI, Wappner RS, Sweetman L: Mevalonic Aciduria — An Inborn Error of Cholesterol and Nonsterol Isoprene Biosynthesis. N Engl J Med 1986;314:1610-1614.

- 4 Cuisset L, Drenth JP, Simon A, Vincent MF, van der Velde Visser S, van der Meer JW, Grateau G, Delpech M, International Hyper-IgD Study Group: Molecular analysis of MVK mutations and enzymatic activity in hyper-IgD and periodic fever syndrome. Eur J Hum Genet EJHG 2001;9:260-266.

-5 Gomez AR, Couce ML, Garcia-Villoria J, Torres A, Souto AB, Yagüe J, Vilaseca MA, Ribes A, Aróstegui JI: Clinical, Genetic, and Therapeutic Diversity in 2 Patients With Severe Mevalonate Kinase Deficiency. Pediatrics 2012;129:e535-e539.

-6 van der Hilst JCH, Bodar EJ, Barron KS, Frenkel J, Drenth JPH, van der Meer JWM, Simon A: Long-Term Follow-Up, Clinical Features, and Quality of Life in a Series of 103 Patients With Hyperimmunoglobulinemia D Syndrome. Medicine (Baltimore) 2008;87:301-310.

7 Goebel-Schreiner B, Schreiner R, Hoffmann GF, Gibson KM, Hoffmann GF, Goebel-Schreiner B, Schreiner R: Segregation of the N301T mutation in the family of the index patient with mevalonate kinase deficiency. J Inherit Metab Dis 1995;18:197-200.

-8 Houten SM, Kuis W, Duran M, de Koning TJ, van Royen-Kerkhof A, Romeijn GJ, Frenkel J, Dorland L, de Barse MM, Huijbers WA, Rijkers GT, Waterham HR, Wanders RJ, Poll-The BT: Mutations in MVK, encoding mevalonate kinase, cause hyperimmunoglobulinaemia D and periodic fever syndrome. Nat Genet 1999;22:175-177.

-9 Hinson DD, Ross RM, Krisans S, Shaw JL, Kozich V, Rolland MO, Divry P, Mancini J, Hoffmann GF, Gibson KM: Identification of a mutation cluster in mevalonate kinase deficiency, including a new mutation in a patient of Mennonite ancestry. Am J Hum Genet 1999;65:327-335.

10 Marcuzzi A, Vozzi D, Girardelli M, Tricarico PM, Knowles A, Crovella S, Vuch J, Tommasini A, Piscianz E, Bianco AM: Putative modifier genes in mevalonate kinase deficiency. Mol Med Rep 2016;13:3181-3189.

$\checkmark 11$ Moura R, Tricarico PM, Campos Coelho AV, Crovella S: GRID2 a novel gene possibly associated with mevalonate kinase deficiency. Rheumatol Int DOI:10.1007/s00296-014-3115-y.

12 Browne C, Timson DJ: In Silico Prediction of the Effects of Mutations in the Human Mevalonate Kinase Gene: Towards a Predictive Framework for Mevalonate Kinase Deficiency. Ann Hum Genet 2015;79:451459.

13 Thurnher M, Gruenbacher G, Nussbaumer O: Regulation of mevalonate metabolism in cancer and immune cells. Biochim Biophys Acta BBA - Mol Cell Biol Lipids 2013;1831:1009-1015.

14 Henneman L, Schneiders MS, Turkenburg M, Waterham HR: Compromized geranylgeranylation of RhoA and Rac1 in mevalonate kinase deficiency. J Inherit Metab Dis 2010;33:625-632.

15 Marcuzzi A, Tricarico PM, Piscianz E, Kleiner G, Vecchi Brumatti L, Crovella S: Lovastatin induces apoptosis through the mitochondrial pathway in an undifferentiated SH-SY5Y neuroblastoma cell line. Cell Death Dis 2013;4:e585.

16 Zanin V, Marcuzzi A, Kleiner G, Piscianz E, Monasta L, Zacchigna S, Crovella S, Zauli G: Lovastatin dosedependently potentiates the pro-inflammatory activity of lipopolysaccharide both in vitro and in vivo. J Cardiovasc Transl Res 2013;6:981-988.

17 Tricarico PM, Piscianz E, Monasta L, Kleiner G, Crovella S, Marcuzzi A: Microglia activation and interaction with neuronal cells in a biochemical model of mevalonate kinase deficiency. Apoptosis Int J Program Cell Death 2015;20:1048-1055.

18 Marcuzzi A, Zanin V, Kleiner G, Monasta L, Crovella S: Mouse model of mevalonate kinase deficiency: comparison of cytokine and chemokine profile with that of human patients. Pediatr Res 2013;74:266-271.

19 Schindelin J, Arganda-Carreras I, Frise E, Kaynig V, Longair M, Pietzsch T, Preibisch S, Rueden C, Saalfeld S, Schmid B, Tinevez J-Y, White DJ, Hartenstein V, Eliceiri K, Tomancak P, Cardona A: Fiji: an open-source platform for biological-image analysis. Nat Methods 2012;9:676-682.

20 Bolte S, Cordelières FP: A guided tour into subcellular colocalization analysis in light microscopy. J Microsc 2006;224:213-232.

21 Sorrentino G, Ruggeri N, Specchia V, Cordenonsi M, Mano M, Dupont S, Manfrin A, Ingallina E, Sommaggio R, Piazza S, Rosato A, Piccolo S, Del Sal G: Metabolic control of YAP and TAZ by the mevalonate pathway. Nat Cell Biol 2014;16:357-366.

22 Kaur J, Debnath J: Autophagy at the crossroads of catabolism and anabolism. Nat Rev Mol Cell Biol $2015 ; 16: 461-472$. 


\section{Cellular Physiology Cell Physiol Biochem 2017;41:1649-1660 \begin{tabular}{ll|l} 
and Biochemistry Published onlIne: IVIarch 28, 2017 & $\begin{array}{l}\text { (C) } 2017 \text { The Author(s). Published by S. Karger AG, Basel } \\
\text { www.karger.com/cpb }\end{array}$
\end{tabular}}

23 Lionaki E, Markaki M, Palikaras K, Tavernarakis N: Mitochondria, autophagy and age-associated neurodegenerative diseases: New insights into a complex interplay. Biochim Biophys Acta DOI:10.1016/j. bbabio.2015.04.010.

24 Kesidou E, Lagoudaki R, Touloumi O, Poulatsidou K-N, Simeonidou C: Autophagy and neurodegenerative disorders. Neural Regen Res 2013;8:2275-2283.

25 Ghavami S, Shojaei S, Yeganeh B, Ande SR, Jangamreddy JR, Mehrpour M, Christoffersson J, Chaabane W, Moghadam AR, Kashani HH, Hashemi M, Owji AA, Łos MJ: Autophagy and apoptosis dysfunction in neurodegenerative disorders. Prog Neurobiol 2014;112:24-49.

26 Vidal RL, Matus S, Bargsted L, Hetz C: Targeting autophagy in neurodegenerative diseases. Trends Pharmacol Sci 2014;35:583-591.

27 Celsi F, Piscianz E, Romano M, Crovella S: Knockdown of MVK does not lead to changes in NALP3 expression or activation. J Inflamm Lond Engl 2015;12:7.

28 Hager EJ, Tse HM, Piganelli JD, Gupta M, Baetscher M, Tse TE, Pappu AS, Steiner RD, Hoffmann GF, Gibson KM: Deletion of a single mevalonate kinase (Mvk) allele yields a murine model of hyper-IgD syndrome. J Inherit Metab Dis 2007;30:888-895.

29 Hager EJ, Piganelli JD, Tse HM, Gibson KM: Aberrant expression of costimulatory molecules in splenocytes of the mevalonate kinase-deficient mouse model of human hyper-IgD syndrome (HIDS). J Inherit Metab Dis 2011;35:159-168.

-30 Celec P, Behuliak M: The lack of non-steroid isoprenoids causes oxidative stress in patients with mevalonic aciduria. Med Hypotheses 2008;70:938-940.

-31 Mariño G, Niso-Santano M, Baehrecke EH, Kroemer G: Self-consumption: the interplay of autophagy and apoptosis. Nat Rev Mol Cell Biol 2014;15:81-94.

-32 Sarkar C, Zhao Z, Aungst S, Sabirzhanov B, Faden AI, Lipinski MM: Impaired autophagy flux is associated with neuronal cell death after traumatic brain injury. Autophagy 2014;10:2208-2222.

-33 Ao X, Zou L, Wu Y: Regulation of autophagy by the Rab GTPase network. Cell Death Differ 2014;21:348358.

-34 Tricarico PM, Crovella S, Celsi F: Mevalonate Pathway Blockade, Mitochondrial Dysfunction and Autophagy: A Possible Link. Int J Mol Sci 2015;16:16067-16084.

-35 Cejka D, Hayer S, Niederreiter B, Sieghart W, Fuereder T, Zwerina J, Schett G: Mammalian target of rapamycin signaling is crucial for joint destruction in experimental arthritis and is activated in osteoclasts from patients with rheumatoid arthritis. Arthritis Rheum 2010;62:2294-2302.

-36 Bruyn G a. W, Tate G, Caeiro F, Maldonado-Cocco J, Westhovens R, Tannenbaum H, Bell M, Forre O, Bjorneboe O, Tak PP, Abeywickrama KH, Bernhardt P, van Riel PLC, RADD Study Group: Everolimus in patients with rheumatoid arthritis receiving concomitant methotrexate: a 3-month, double-blind, randomised, placebo-controlled, parallel-group, proof-of-concept study. Ann Rheum Dis 2008;67:10901095.

-37 van der Burgh R, Pervolaraki K, Turkenburg M, Waterham HR, Frenkel J, Boes M: Unprenylated RhoA contributes to IL-1 $\beta$ hypersecretion in mevalonate kinase deficiency model through stimulation of Rac1 activity. J Biol Chem 2014;289:27757-27765.

38 Jurczyluk J, Munoz MA, Skinner OP, Chai RC, Ali N, Palendira U, Quinn JM, Preston A, Tangye SG, Brown AJ, Argent E, Ziegler JB, Mehr S, Rogers MJ: Mevalonate kinase deficiency leads to decreased prenylation of Rab GTPases. Immunol Cell Biol DOI:10.1038/icb.2016.58.

- 39 Veluthakal R, Arora DK, Goalstone ML, Kowluru RA, Kowluru A: Metabolic Stress Induces Caspase-3 Mediated Degradation and Inactivation of Farnesyl and Geranylgeranyl Transferase Activities in Pancreatic $\beta$-Cells. Cell Physiol Biochem 2016;39:2110-2120. 